\title{
Design and implementation of network streaming media play system based on DSP
}

\author{
Jinhai Zhang, Hongbing Qin \\ Marine college of Shandong jiaotong university, Weihai , Shandong,China
}

Keywords: Streaming media, network, digital signal processor, frame of reference standards, DSP

\begin{abstract}
With the development of multimedia technology and the popularity of Internet technologies, network streaming media of communication have become multimedia communication is required. Using existing audio and video encoding and decoding algorithm can dramatically reduce the amount of data transferred, and reduces network bandwidth requirements, but due to the application of network stream media real time tend to be high, so real time implementation of the codec has become a key issue. Using embedded processor core processor method of combining hardware and software has become an effective realization of the video codec.
\end{abstract}

\section{Introduction}

With the Internet on a global scale of popularization and development of video codec technology, digital video technology has been widely used, in particular, the appearance of new multimedia services, such as video conferencing, video on demand, remote monitoring, such as HDTV, based on Internet and video coding for video transmission systems become one of the top current information field.

Development of video codec technology provides the basis for Internet video communications guaranteed. As the amount of video data than audio, uncompressed video in CIF format, 25 frames per second would be 3.8Mbyte the amount of data, the bandwidth and storage space is limited Internet and wireless network of mobile networks for data transmission is not realistic, and the rapid development of video codec makes it possible. As early as 1986, ITU proposed the H.261 standard, went on to launch standards such as JPEG, MPEG-4. The current most widely applied to H.263 video transmission in a network, and the latest MPEG-4, H.264 standard of performance is excellent.

How to ensure real time video transmission system has been the one of the key issues. Pure software system is easy to upgrade and migration, but poor live performance special codec chip can guarantee real-time performance, but the system substantially less flexibility, does not favor the algorithm upgrade. IT designed for multimedia applications the DM642 chip solved these problems, which makes it almost is a multimedia-rich peripheral interface single-chip hardware platforms for embedded systems, its complete programmability, also makes it compatible with evolving multimedia signal processing standards constitute a common software platform. Using embedded processor core processor method of combining software and hardware video codec has been an effective approach.

\section{System development platform}

Dedicated to video and multimedia application development the image processor DM642 is currently building a good platform for multimedia communication systems. It makes it almost is a multimedia-rich peripheral interface single-chip hardware platforms for embedded systems, its complete programmability makes it compatible with evolving multimedia signal processing standards, to form a common software platform.

In order to meet the needs of video and image processing, the series DSP architecture. The advanced structure of very long instruction word, in one instruction cycle to process multiple instructions in parallel. 


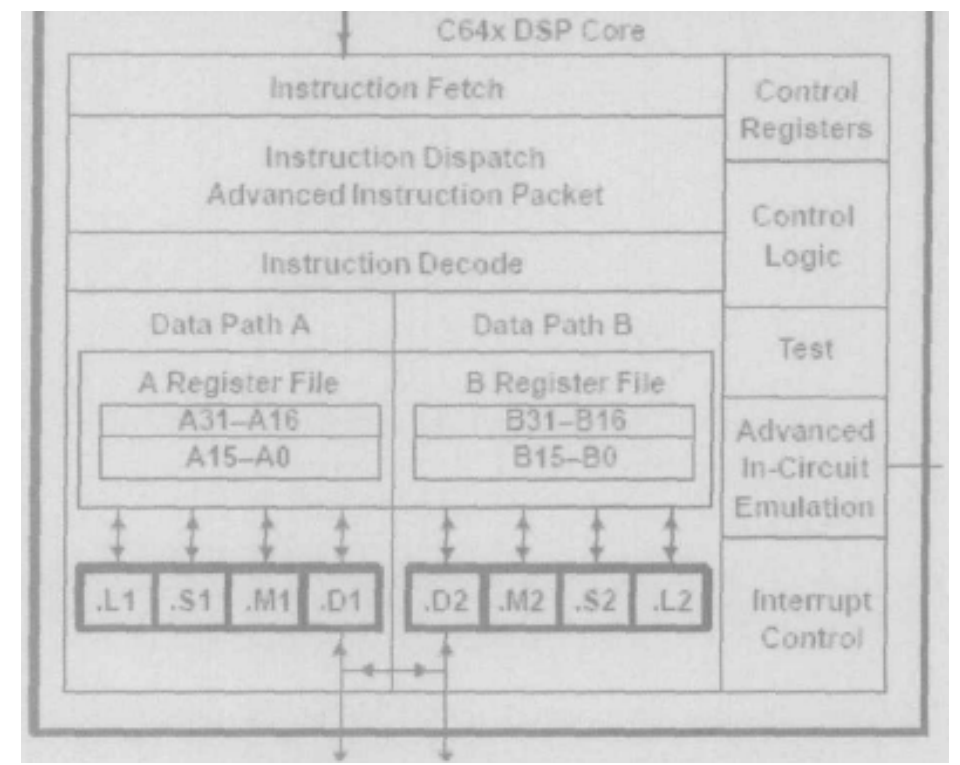

Fig.1. DM642 CPU chart

Most major features was adopted in very long instruction word, structure. In of system structure in the by a super long of machine instruction word to drive internal of 8 a function unit, each instruction word contains has 8 a field instruction, field Zhijian mutual independent, their control a function unit, so can in single cycle within launches out more article instruction, achieved is high of instruction level parallel efficiency, up can in single cycle within while implementation 8 article instruction. Compile assembler compiler process, decide which instruction in the code into a very long machine instructions, in a period of parallel execution. This instruction on the parallel arrangement is static, which means that all instruction is determined at compile time, once decided, then, regardless of any run, it remains unchanged.

In order to solve the bandwidth between the CPU and memory, memory architecture and design becomes important, and memory architecture is the design principal contradiction in memory capacity and contradictions between the requirements of speed and cost. Fast on-chip memory, small capacity, high costs, external memory capacity, low cost, speed and, therefore, the multi-level storage architecture.

RF5 is a frame of reference for great scalability, which design will enable designers to create complex algorithms based on multi-channel applications. Entirely written in c code can be easily transplanted and modified the framework makes developers can associate one or more frameworks as a basis for your own applications. Scalable algorithm for channel management feature makes the program contains a large number of tasks while using as few threads algorithm able to efficiently share data between memory and can be easily replaced by ICELL interface algorithm. SCOM module can help with the efficient communication between tasks for easy multitasking multi-threaded application development. These features from the underlying complexity of the design, and will make the software framework is more widely used.

Rate control method is the use of the simplest fixed frame Skip method, that is, each encoding a frame skip a few frames, this method is easy to implement, you can secure a stable frame rate, but cannot get a steady rate. In need of strict control of bit-rate transmission channel, PSTN network or wireless network bandwidth-limited channel requires constant bit-rate control method. To this end, the algorithm uses an Adaptive rate control method.

\section{ActiveX and Web communication method}

ActiveX control properties, methods, and events are Activex controls three ways to communicate with its container, container for the student's application and convenient operation the student insert ActiveX control into a Web page, and class live in the same interface, which gives a simple and comfortable feeling. But then came a problem: in students and teachers interact in the process, will also have a live voice and real-time interactive voice, live sound using MMS protocols published, 
there will be a 15-20-second delay, so real-time interaction with the sound out of sync, how do we solve this problem? The simplest is the most practical method is closed when the demand for real-time interaction is live Media Player plug-in in a Web page's voice, in interaction after the open sound. But the two plug-ins are not able to communicate directly, needs to have a the middle of a communication object. The communication object is IE container in the middle (or other container).

A typical includes design-time and run-time user interface, only one IDispatch interface defines the methods and properties of the control, only the IConnectionPoint interface is used to control events that are raised. In addition, one can also contain the whole life cycle of a medical support, as well as clip art, user interface features such as drag-and-drop support.

From the structural point of view, a control a large number of COM interface must support in order to use these features. Active x controls are always placed in the containers they placed in the running, the extension of the control. OCX, but from the perspective of running modules, it is a standard WINDOWS DLL file.

Properties and methods is the way applications communicate with the control within the container, containers and controls communications between events. For ActiveX controls, the IDispatch interface of the event is to end when the container. The underlying mechanism of the event called a connection point. A connection point is required to communicate with the container interface type description, connection points are not limited to IDispatch interface, it can also be used in any COM implementation, control only the first using them.

\section{System design of network functions}

Application process application layer directly to the users of services, determine the nature of communication between processes to meet the needs of users.

The transport layer is responsible for communication between two processes in the host, data transmission units for the segment. The layers have reuse and sharing capabilities. Multiple processes in the re with the following network transmission function, to the destination host's network layer, and then use the function, to deliver data to the corresponding process.

Select the appropriate routing network layer, passed down from the transport layer of the sending station grouped according to find the destination host.

Data link layer in the network's two adjacent nodes on the lines of error-free transmission of data, in frames and put a real link that can go wrong, looking down into the network layer as if it were a link that does not make mistakes.

Physical layer of transparent transmission of bit-stream.

Application layer is the highest principle of architecture layer, application layer above there are no other layer, use of upper tier's mission is not to provide services, but provide services directly to the users of the application process, and communicate with each other to solve a specific application process is known as the "application process".

UDP protocol is a protect message boundaries, does not guarantee the reliability of data transfer, support bi-directional data flow, but is not guaranteed to be reliable, ordered, duplicate-free. For the transmission of digital video signal, select the appropriate transport protocol should be based on the actual situation to meet the needs of video data transmission. Digital video signal very large amounts of data, if the TCP protocol transport is bound to consume too much network resources, and cannot be guaranteed real-time performance, and uses the UDP protocol is more in line with the demands of real time monitoring system. Transmission, broadcasting information such as real-time voice, video, and so on.

System's hardware consists of both the server and client parts. In the servers section, single-channel video input signal acquisition is realized, and sent through the network interface of the encoded stream, network video encoder. System structure as shown in Figure 2.Completed the video stream on the client to receive, decode and display. First data stream received through the network interface, and H.263 decoder receives encoded streams, and finally through the DM642 output of a channel. 


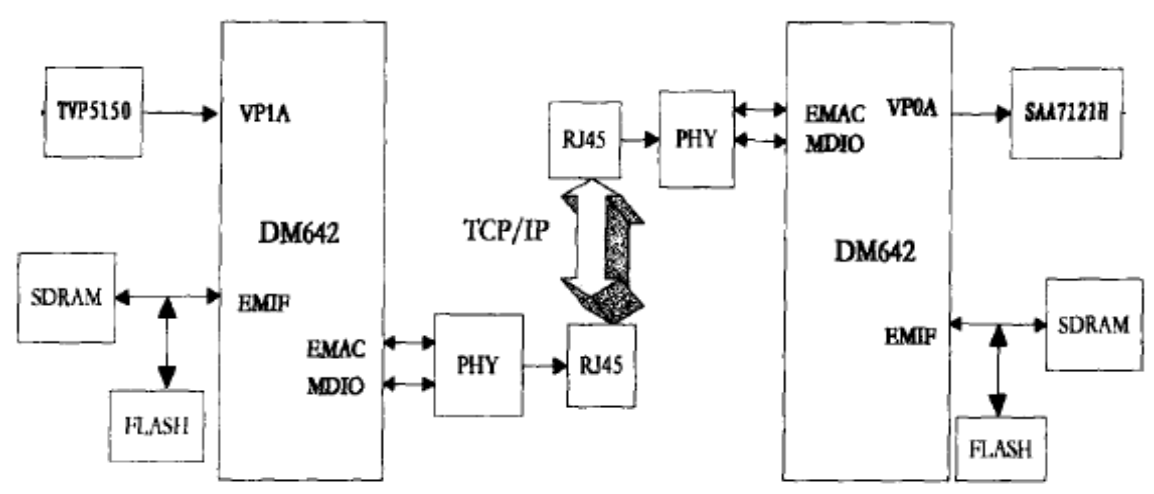

Fig.2. System architecture diagram

This real-time interaction will result in different steps, this time lag is less likely, because real-time interaction must be real-time, media must be buffered through streaming media server release. Now all I have to do is to select different audio, screen out Media audio effects, video synchronization problem has no solution.

In actual development and test process in the met many of problem, as communications in the due to network status not stable (especially public network communications) caused sound video play not smooth; both inside and outside network between points on points communications of problem; database shared of problem; also has site live and real-time interactive synchronization communications of problem, these problem through check out related information and himself for analysis and research found some feasible of method, in actual application in the can better of operation. There are some issues such as teacher's desktop collection and synchronization problems with the interaction has not yet found the perfect solution, I hope in future studies to these issues in depth.

The topic's significance lies in modern teaching needs, starting from the network stream media technology and design which are consistent with the characteristics of modern teaching live interactive teaching system in real time. While Internet streaming media and some knowledge in network communication system for induction and consolidation. Through the study of real-time live interactive teaching system analysis, able to explain the basic development of stream media communication processes.

\section{References}

[1] Gao Wen, multimedia data compression technology, the Beijing Publishing House of electronics industry, 2009

[2] Lu Shiwen. Principles of computer networks and network technology. China machine press, 2006

[3] Hill R,Wang J,Nahrstedt K.Quantifying Non-functional Requirements:A Process Oriented Approach[C]. IEEE International Requirements Engineering Conference, Kyoto, Japan, 2004: 352-353

[4] Bryan Ford, Pyda Srisuresh, and Dan Kegel. Peer-to-Peer Communication Across Network Address Translators. In USENIX Annual Technical Conference, Anaheim, 2005.4

[5] Lu Qiming. Windows Media Program Guide., Tsinghua University Press, 2005

[6] Shi Donghui, Cai Qingsheng, Ni Zhiwei. disaggregated data on methods of outliers mining based on rule research. of computer research and development 2007.

[7] Ming Fan, Fan Hongjian. Introduction to data mining. the people's posts and telecommunications Publishing House 2006. 